ARTICLE

\title{
Efficient and large-area all vacuum-deposited perovskite light-emitting diodes via spatial confinement
}

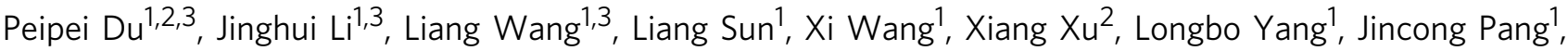
Wenxi Liang${ }^{1}$, Jiajun Luo (1) ${ }^{1 凶}$, Ying Ma (i] ${ }^{2} \&$ Jiang Tang (i) ${ }^{1 凶}$

With rapid advances of perovskite light-emitting diodes (PeLEDs), the large-scale fabrication of patterned PeLEDs towards display panels is of increasing importance. However, most state-of-the-art PeLEDs are fabricated by solution-processed techniques, which are difficult to simultaneously achieve high-resolution pixels and large-scale production. To this end, we construct efficient $\mathrm{CsPbBr}_{3}$ PeLEDs employing a vacuum deposition technique, which has been demonstrated as the most successful route for commercial organic LED displays. By carefully controlling the strength of the spatial confinement in $\mathrm{CsPbBr}_{3}$ film, its radiative recombination is greatly enhanced while the nonradiative recombination is suppressed. As a result, the external quantum efficiency (EQE) of thermally evaporated PeLED reaches $8.0 \%$, a record for vacuum processed PeLEDs. Benefitting from the excellent uniformity and scalability of the thermal evaporation, we demonstrate PeLED with a functional area up to 40.2 $\mathrm{cm}^{2}$ and a peak EQE of $7.1 \%$, representing one of the most efficient large-area PeLEDs. We further achieve high-resolution patterned perovskite film with $100 \mu \mathrm{m}$ pixels using fine metal masks, laying the foundation for potential display applications. We believe the strategy of confinement strength regulation in thermally evaporated perovskites provides an effective way to process high-efficiency and large-area PeLEDs towards commercial display panels.

\footnotetext{
${ }^{1}$ Wuhan National Laboratory for Optoelectronics (WNLO) and School of Optical and Electronic Information, Huazhong University of Science and Technology (HUST), Wuhan, Hubei, China. ${ }^{2}$ State Key Laboratory of Materials Processing and Die \& Mould Technology, School of Materials Science and Engineering, Huazhong University of Science and Technology (HUST), Wuhan, Hubei, China. ${ }^{3}$ These authors contributed equally: Peipei Du, Jinghui Li, Liang Wang.

凶email: luojiajun@mail.hust.edu.cn; jtang@mail.hust.edu.cn
} 
T he ubiquity of display technology in our day-to-day lives brings a trillion-dollar market worldwide, great efforts thus have been directed towards the development of highperformance and cost-effective display technologies. The recently emerged perovskite light-emitting diodes (PeLEDs) have attracted numerous interests in both academic and industrial fields, due to their high efficiency, narrow emission linewidth as well as low costs of material and fabrication ${ }^{1-5}$. Specifically, the CIE coordinates of PeLEDs could reach $140 \%$ of the NTSC standard 6 , which is much wider than those of industrially dominant liquid crystal displays (LCDs) and organic light-emitting diodes (OLEDs) technologies, and the raw material costs of PeLEDs are much cheaper than those of OLEDs. Since the first report of PeLEDs in 2014 by Tan and co-workers ${ }^{7}$, substantial progress has been made in the past several years, achieving an external quantum efficiency (EQE) greater than $20 \%$, which is comparable with that of the best-performing OLEDs $^{8-10}$. These breakthroughs represent an encouraging step towards the practical application of PeLEDs for next-generation display technologies.

For a full-color display, blue, green, and red-emitting pixels with well-designed size and shape are periodically patterned, which are driven by the thin-film transistors (TFT) underneath. The manufacturing of display panels thus relies critically on the integration of large-scale perovskite micro-arrays and TFT panels. Currently, most PeLEDs are fabricated by solution-processed methods with the best performance achieved in functional areas below $0.1 \mathrm{~cm}^{2},{ }^{11}$. One impressive exception is reported by Wang et al. who demonstrated a high EQE of over $16 \%$ for their spincoated PeLED employing a molecular modification strategy ${ }^{12}$. A further scale-up using blade-coating enables an ultra-large PeLED with an area of $4 \mathrm{~cm} \times 7 \mathrm{~cm}$; however, no efficiency was reported for this PeLED ${ }^{13}$. It should also be noted that both spin-coating ${ }^{14}$ and blade-coating are difficult to integrate red, green, and blue perovskite pixels on the pre-pixelated TFT panels with high resolution. Inkjet printing patterned PeLEDs may offer one possible solution, however, they show inferior device performance with a maximum $\mathrm{EQE}$ of $3 \%$ due to nonideal film quality and "coffee ring" effect ${ }^{15,16}$. Thereby, the state-of-the-art solutionprocessed PeLEDs remain a significant gap towards full-color display applications. It is thus of urgent importance to develop rational strategies to manufacture efficient and large-area patterned PeLEDs for next-generation display technologies.

Thermal evaporation has been widely used in the semiconductor industry due to its remarkable scalability and reproducibility, as successfully demonstrated by commercial $\mathrm{OLEDs}^{17,18}$. Side-by-side three-color perovskite patterns can be easily achieved through sequent deposition with the assistance of fine metal masks, which resemble that of OLEDs. It can be expected that the mature evaporation process and facilities can promote the fabrication of PeLED display panels (Supplementary Table 1) ${ }^{19,20}$. Notably, thermal evaporation is free of toxic solvent and particularly advantageous for depositing hardly soluble inorganic perovskites, such as $\mathrm{CsPbX}_{3}(\mathrm{X}=\mathrm{Cl}, \mathrm{Br}, \mathrm{I})^{21}$. Besides, thermal evaporation has been successfully demonstrated by perovskite photovoltaics with power conversion efficiency beyond $20 \%$, suggesting high film quality via thermal evaporation ${ }^{22}$.

Despite holding great promises, the explorations on thermally evaporated PeLEDs are very limited, and their device performance lags significantly behind, which calls for further improvements ${ }^{11}$. In thermally evaporated $\mathrm{CsPbBr}_{3}$ films, the three dimensional (3D) network of corner-sharing $\left[\mathrm{PbX}_{6}\right]^{4-}$ octahedra enables large band dispersion and small exciton binding energy at room temperature, indicating that the excitons readily dissociate into free carriers, which are easily captured by nonradiative recombination centers and result in poor photoluminescence 23,24 . Previous studies have demonstrated the most efficient way to solve this problem is by designing lowdimensional or multiple quantum well structures in perovskite emitters, aiming to enhance radiative recombination rate by strongly confining the electrons and holes ${ }^{25-27}$. However, a fundamental study of the spatial confinement, the associated recombination dynamics, and device behavior in the $\mathrm{Cs}-\mathrm{Pb}-\mathrm{Br}$ system, which can provide useful guidelines for the optimization of thermally evaporated PeLEDs, is still lacking ${ }^{23,28}$.

Herein, we report the development of efficient and large-area PeLEDs based on vacuum-deposited $\mathrm{Cs}-\mathrm{Pb}-\mathrm{Br}$ films via spatial confinement. By incorporating zero-dimensional (0D) structural $\mathrm{Cs}_{4} \mathrm{PbBr}_{6}$ into $\mathrm{CsPbBr}_{3}$ film, the $3 \mathrm{D}$ connection of $\left[\mathrm{PbBr}_{6}\right]^{4-}$ octahedra is partially broken, forming $\mathrm{Cs}_{4} \mathrm{PbBr}_{6} / \mathrm{Cs} \mathrm{PbBr}_{3}$ coreshell structure. The electrons and holes formed in $\mathrm{CsPbBr}_{3}$ are spatially confined by $\mathrm{Cs}_{4} \mathrm{PbBr}_{6}$. As expected, a blue shift in photoluminescence (PL) wavelength from $516-480 \mathrm{~nm}$ is observed as the $\mathrm{CsBr} / \mathrm{PbBr}_{2}$ molar ratio increased from 1.24-2.28. We then further investigated their carrier recombination dynamics via femtosecond-transient absorption and found that perovskite film of $\mathrm{CsBr} / \mathrm{PbBr}_{2}=1.56$ shows enhanced radiative recombination rate and suppressed nonradiative recombination rate compared to that of $\mathrm{CsBr} / \mathrm{PbBr}_{2}=1.24$. Based on an optimal molar ratio of $\mathrm{CsBr} / \mathrm{PbBr}_{2}$, we then constructed the most efficient thermally evaporated PeLED with a record EQE of $8.0 \%$. Moreover, we successfully fabricated large-area $(10 \mathrm{~cm} \times 9 \mathrm{~cm})$ and patterned perovskite films (the diameter of pixels is $\sim 100 \mu \mathrm{m}$ ), emphasizing the great superiority of our thermal evaporation process. More importantly, we achieved a 7.1\% EQE for the 40.2 $\mathrm{cm}^{2}$ device, representing one of the most efficient large-area PeLEDs. We believe our works represent an encouraging stepping stone to bring state-of-the-art PeLEDs from the laboratory towards commercial display panels.

\section{Results}

Manipulation of spatial confinement in thermally evaporated Cs-Pb-Br film. $\mathrm{Cs} \mathrm{PbBr}_{3}$ has been extensively investigated as a promising material for light-emitting applications, however, the $3 \mathrm{D}$ connection of $\left[\mathrm{PbBr}_{6}\right]^{4-}$ gives rise to large band dispersion, enables excitons to dissociate readily, and leads to a slow radiative recombination rate ${ }^{29,30}$. Organic ligands, ammonium halides with large organic cations or polymer matrixes are commonly introduced to enhance radiative recombination rate by providing spatial confinement of electrons and holes ${ }^{28,31,32}$, however, they suffer from low thermal stability due to their organic nature. Figure 1a shows the phase diagram of the $\mathrm{CsBr}-\mathrm{PbBr}_{2}$ binary system, highlighting not only $\mathrm{CsPBBr}_{3}$ but also $\mathrm{CsPb}_{2} \mathrm{Br}_{5}$ and $\mathrm{Cs}_{4} \mathrm{PbBr}_{6}$ are thermodynamically stable phases that can be synthesized based on the different molar ratios of $\mathrm{CsBr} / \mathrm{PbBr}_{2}{ }^{33}$. The Cs-rich phase $\mathrm{Cs}_{4} \mathrm{PbBr}_{6}$ possesses a 0D structure with $\left[\mathrm{PbBr}_{6}\right]^{4-}$ octahedra completely isolated by $\mathrm{Cs}^{+}$cations (Fig. $\left.1 \mathrm{~b}\right)^{34}$, which can serve as a barrier to break the connectivity of $\left[\mathrm{PbBr}_{6}\right]^{4-}$ in $\mathrm{Cs} \mathrm{PbBr}_{3}$. Figure $1 \mathrm{~b}$ shows the crystal model of $\mathrm{Cs} \mathrm{PbBr}_{3} / \mathrm{Cs}_{4} \mathrm{PbBr}_{6}$ composites. $\mathrm{CsPbBr}_{3}$ can be viewed as nano-inclusions embedded in the $\mathrm{Cs}_{4} \mathrm{PbBr}_{6}$ matrix, which resemble II-VI, IV-VI, and III-V quantum dots and the size of $\mathrm{CsPbBr}_{3}$ nano-inclusions determines the strength of quantum confinement effect ${ }^{35}$. Thanks to no intermediate phase between $\mathrm{Cs}_{3} \mathrm{PBr}_{3}$ and $\mathrm{Cs}_{4} \mathrm{PbBr}_{6}$ in the phase diagram (Fig. 1a), the molar ratio of $\mathrm{CsBr}$ and $\mathrm{PbBr}_{2}$ precursors directly determines the proportions of $\mathrm{CsPbBr}_{3}$ and $\mathrm{Cs}_{4} \mathrm{PbBr}_{6}$. Thereby, the strength of the confinement can be controlled by the molar ratio of $\mathrm{CsBr} / \mathrm{PbBr}_{2}$ instead of introducing foreign organic additives, enabling higher thermal stability 
a

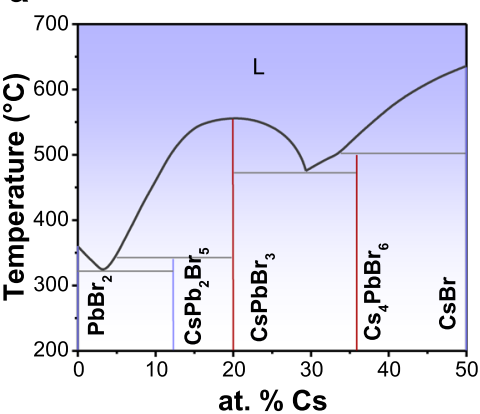

d

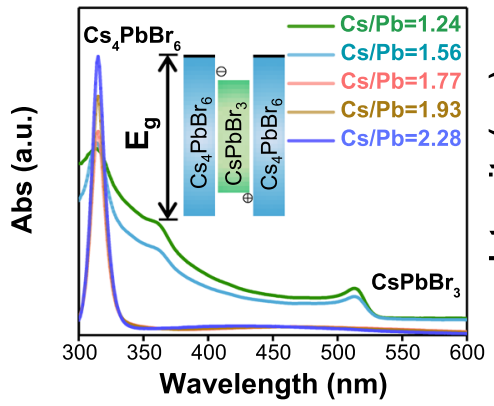

b

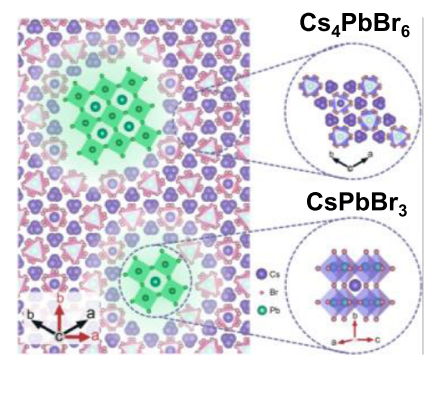

C

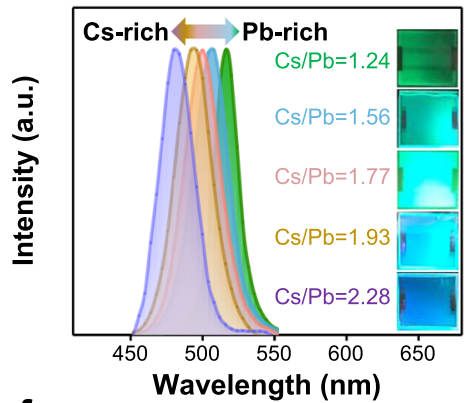

f
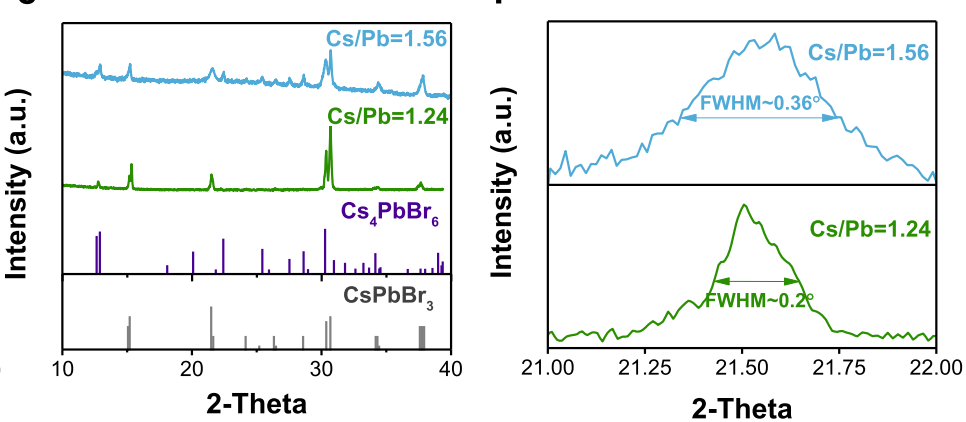

Fig. 1 Characterizations of thermally evaporated $\mathbf{C s}-\mathbf{P b}-\mathbf{B r}$ film. a The phase diagram of $\mathrm{CsBr}_{\mathbf{m}}-\mathrm{PbBr}_{2}$ binary system, which is provided under licence from the online database of SpringerMaterials ${ }^{49}, 50$. b The schematic diagram of $\mathrm{CsPbBr}_{3}$ embedded in the $\mathrm{Cs}_{4} \mathrm{PbBr} \mathrm{Pb}_{6}$ matrix, which indicates the $3 \mathrm{D}\left[\mathrm{PbBr}_{6}\right]^{4-}$ of $\mathrm{CsPbBr}_{3}$ is separated by $\mathrm{OD}\left[\mathrm{PbBr}_{6}\right]^{4-}$ of $\mathrm{Cs}_{4} \mathrm{PbBr}_{6}$. c, d The $\mathrm{PL}$ (c) and absorption (d) spectra of $\mathrm{Cs}-\mathrm{Pb}-\mathrm{Br}$ films with various $\mathrm{Cs} / \mathrm{Pb}$ ratios. e, $\mathbf{f}$ XRD (e) and its magnification (f) for the film of $\mathrm{Cs} / \mathrm{Pb}=1.56$ and $\mathrm{Cs} / \mathrm{Pb}=1.24$

compared to organic-inorganic hybrid perovskites (Supplementary Fig. 1). Moreover, the lattice constant of $\mathrm{Cs}_{4} \mathrm{PbBr}_{6}$ matches well with that of $\mathrm{CsPbBr}_{3}$, indicating that $\mathrm{Cs}_{4} \mathrm{PbBr}_{6}$ can effectively passivate the surface of $\mathrm{CsPbBr}_{3}$ without additional interface strain $^{35}$.

We thus focus on $\mathrm{Cs}_{4} \mathrm{PbBr}_{6} / \mathrm{CsPbBr}_{3}$ composite films for potential PeLED application. As shown in Fig. 1c, a series of Cs$\mathrm{Pb}-\mathrm{Br}$ films with different $\mathrm{CsBr} / \mathrm{PbBr}_{2}$ molar ratios are fabricated using the thermal co-evaporation (Supplementary Fig. 2), high evaporation rate (Supplementary Fig. 3 and Supplementary Note 1) and low substrate temperature are believed to decrease grain size, further improving spatial confinement (detail in Method section $)^{36,37}$. The $\mathrm{CsBr} / \mathrm{PbBr}_{2}$ ratio is approximately estimated by an energy-dispersive spectrometer (EDS) measurement (Supplementary Table 2). According to the phase diagram, a high $\mathrm{CsBr} / \mathrm{PbBr}_{2}$ ratio generally yields high content of $\mathrm{Cs}_{4} \mathrm{PbBr}_{6}$, probably resulting in small-sized $\mathrm{CsPbBr}_{3}$ nano-inclusion. The emission color of as-prepared $\mathrm{Cs}-\mathrm{Pb}-\mathrm{Br}$ films varies from green to cyan with the PL peak blue-shifts from $516-480 \mathrm{~nm}$ as the ratio of $\mathrm{CsBr} / \mathrm{PbBr}_{2}$ increases from 1.24-2.28. These results match well with the size-dependent band gap of $\mathrm{CsPbr}_{3}$ nano-inclusions, indicating the enhancement of quantum confinement strength ${ }^{35}$. The absorption spectra also reflect the proportion change of $\mathrm{Cs}_{4} \mathrm{PbBr}_{6} / \mathrm{CsPbr}_{3}$ composite films (Fig. 1d). Two absorption peaks located at 315 and $515 \mathrm{~nm}$ are attributed to $\mathrm{Cs}_{4} \mathrm{PbBr}_{6}$ and $\mathrm{Cs} \mathrm{PbBr}_{3}$, respectively. As the $\mathrm{CsBr} / \mathrm{PbBr}_{2}$ precursor ratio increased, the absorption peak from the $\mathrm{Cs}_{4} \mathrm{PbBr}_{6}$ matrix gradually increased while the absorption peak from the $\mathrm{CsPbr}_{3}$ nano-inclusion gradually disappeared, which is consistent with the decreased yellow color in these films (Supplementary Fig. 4). Note that Type-I band alignment of $\mathrm{Cs}_{4} \mathrm{PbBr}_{6} / \mathrm{CsPbBr}_{3}$ facilitates better confinement of carriers in $\mathrm{CsPbBr}_{3}$ with a small bandgap (Fig. 1d, inset $)^{38}$. Due to the moisture instability of $\mathrm{Cs}-\mathrm{Pb}-\mathrm{Br}$ films with high $\mathrm{CsBr} / \mathrm{PbBr}_{2}$ ratios $(\mathrm{Cs} / \mathrm{Pb}=2.28,1.93,1.77)$ (Supplementary Fig. 5), we mainly focus on $\mathrm{Cs}-\mathrm{Pb}-\mathrm{Br}$ films with $\mathrm{CsBr} / \mathrm{PbBr}_{2}$ ratio of 1.56 and 1.24 (named as $\mathrm{Cs} / \mathrm{Pb}=1.56$ and $\mathrm{Cs} / \mathrm{Pb}=1.24$ ) for further characterization. As shown in Fig. 1e, X-ray diffraction (XRD) patterns reveal the coexistence of $\mathrm{Cs} \mathrm{PbBr}_{3}$ and $\mathrm{Cs}_{4} \mathrm{PbBr}_{6}$. Not surprisingly, the $\mathrm{Cs} / \mathrm{Pb}=1.56$ sample exhibits a stronger $\mathrm{Cs}_{4} \mathrm{PbBr}_{6}$ diffraction peak compared to that of $\mathrm{Cs} / \mathrm{Pb}=1.24$, due to the larger presence of $\mathrm{Cs}_{4} \mathrm{PbBr}_{6}$ within the film. To provide direct evidence for the size change of $\mathrm{CsPbBr}_{3}$ nano-inclusion, we magnify the $21.5^{\circ}$ peak, which corresponds to the (110) diffraction peak of $\mathrm{CsPbBr}_{3}$ (Fig. 1f). According to the Debye-Scherrer formula $(D=K \lambda / B \cos \theta$, where $B$ is the FWHM and $D$ is the mean diameter of the crystal domains $)^{32}$, the relatively broader full width at half maximum (FWHM) in the $\mathrm{Cs} / \mathrm{Pb}=1.56$ sample indicates the reduced size of $\mathrm{CsPbBr}_{3}$ nano-inclusions, which is consistent with optical characterizations.

The effect of spatial confinement on the carrier recombination dynamics. Carrier recombination dynamics play a decisive role in emission behavior. We firstly investigate the excitation-densitydependent PL intensity for determining the carrier recombination types. The initial PL intensity $\left(I_{P L}\right)$ shows a quadratic dependence of excited carrier density for the films of $\mathrm{Cs} / \mathrm{Pb}=1.56$ and $\mathrm{Cs} / \mathrm{Pb}$ $=1.24$ (Supplementary Fig. 6), which indicates the radiative recombination process is bimolecular. Meanwhile, by fitting the Arrhenius equation, the exciton binding energies of $\mathrm{Cs} / \mathrm{Pb}=1.56$ and $\mathrm{Cs} / \mathrm{Pb}=1.24$ are derived to be 48.7 and $41.6 \mathrm{meV}$, respectively (Supplementary Fig. 7) 24,37 . The relatively small exciton binding energies close to the room-temperature thermal energy indicate the dominant recombination mechanism is freecarriers $^{39}$. Thereby, the radiative efficiency $\eta$, i.e. photoluminescence quantum yield (PLQY), could be expressed by the 


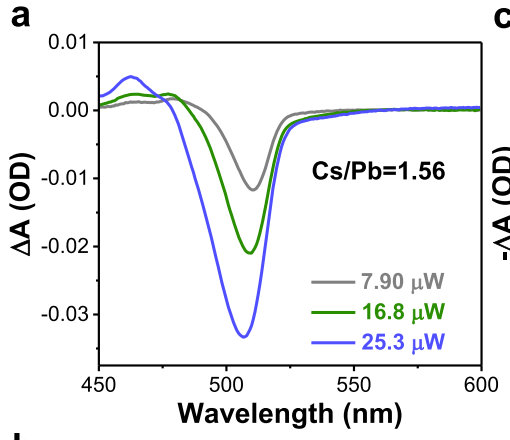

b

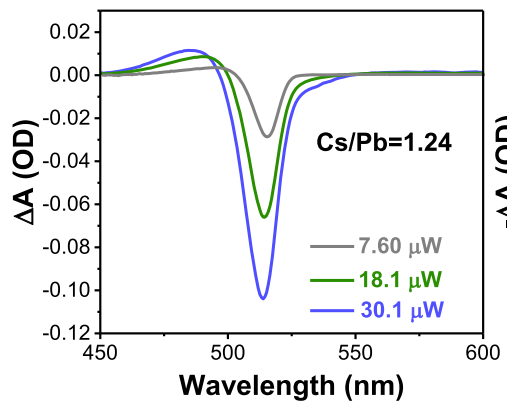

C

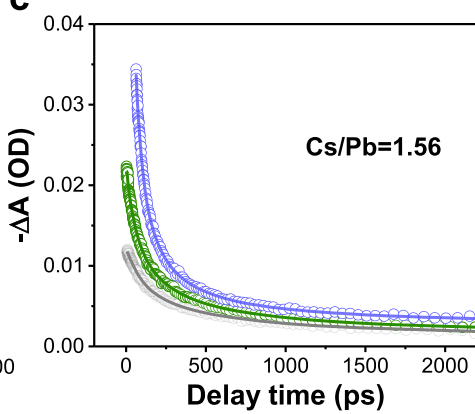

d

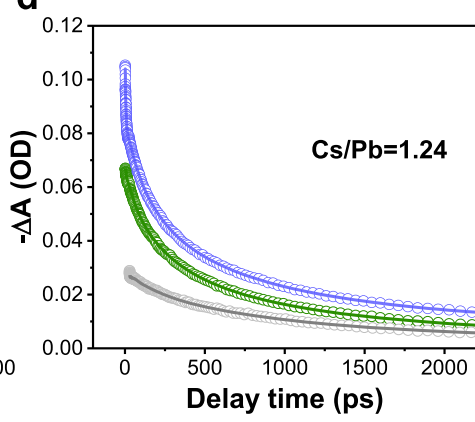

e

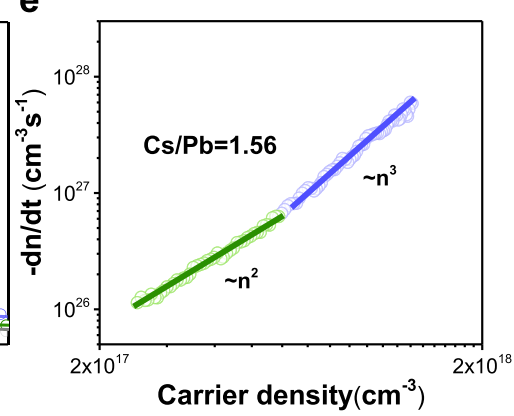

f

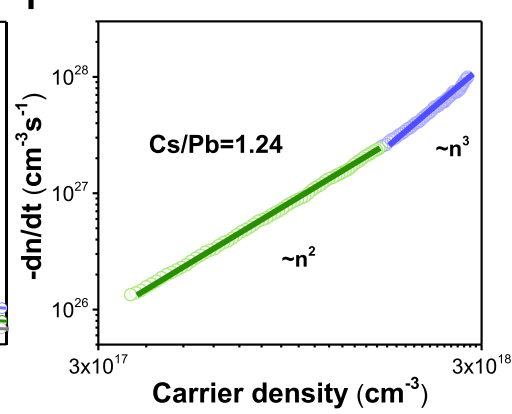

Fig. 2 The recombination dynamics of thermally evaporated Cs-Pb-Br films. a, $\mathbf{b}$ The transient absorption spectra of $\mathrm{Cs} / \mathrm{Pb}=1.56(\mathbf{a})$ and $\mathrm{Cs} / \mathrm{Pb}=1.24$ (b) under $1 \mathrm{ps}$ delay time with various excitation densities. c, d The corresponding dynamic curves of $\mathrm{Cs} / \mathrm{Pb}=1.56(\mathbf{c})$ and $\mathrm{Cs} / \mathrm{Pb}=1.24(\mathbf{d})$. e, $\mathbf{f} \mathrm{The}$ relationship between carrier recombination decay rates and carrier density in the cases of $\mathrm{Cs} / \mathrm{Pb}=1.56(\mathbf{e})$ and $\mathrm{Cs} / \mathrm{Pb}=1.24(\mathbf{f})$. The detailed fitting method is described in the Supplementary Information.

following Eq. 123,28.

$$
\eta=\frac{k_{2} n^{2}}{k_{1} \mathrm{n}+k_{2} n^{2}+k_{3} n^{3}}
$$

where $n$ is the carrier density; $k_{1}$ is the mono-molecular recombination constant, representing trap-assisted recombination; $k_{2}$ is the bi-molecular recombination constant, representing radiative recombination; $k_{3}$ is the tri-molecular recombination constant, representing the nonradiative Auger recombination. As shown in Eq. 1, the radiative efficiency $\eta$ is dependent on the three recombination constants $\left(k_{1}, k_{2}, k_{3}\right)$ and carrier density $(n)$. In the case of extremely low carrier density, trap-assisted recombination dominates and largely determines the PLQY. As the carrier density increases, the radiative recombination starts to compete over trap-assisted recombination and gradually dominates, thus enhancing PLQY. Under the situation of extremely high carrier density, Auger recombination becomes dominative and decreases $\mathrm{PLQY}^{23}$. It is comprehensible that enhancing the radiative recombination rate (larger $k_{2}$ ) and suppressing the nonradiative recombination rate (smaller $k_{1}$ and $k_{3}$ ) could guarantee a higher emissive efficiency.

To quantitatively analyze the recombination dynamics of thermally evaporated $\mathrm{Cs}-\mathrm{Pb}-\mathrm{Br}$ films with different confinement strength, we firstly investigate the $k_{1}$ through time-resolved photoluminescence (TRPL) under extremely low excitation power (Supplementary Fig. 8) where the trap-assisted recombination dominates and high-order recombination contribution is negligible ${ }^{30}$. Consequently, the film of $\mathrm{Cs} / \mathrm{Pb}=1.56\left(k_{1}: 7.14 \times\right.$ $10^{8} \mathrm{~s}^{-1}$ ) possesses a lower trap-assisted nonradiative recombination rate than $\mathrm{Cs} / \mathrm{Pb}=1.24\left(k_{1}: 1.69 \times 10^{9} \mathrm{~s}^{-1}\right)$, attributable to the better interface passivation as more $\mathrm{Cs}_{4} \mathrm{PbBr}_{6}$ presented in the $\mathrm{Cs} / \mathrm{Pb}=$ 1.56 sample $^{40}$. To strengthen our conclusion, we directly characterize the defect densities using space-charge limited current (SCLC) measurement with a device structure of ITO/PEDOT/ perovskite/TAPC/ $\mathrm{MoO}_{3} / \mathrm{Al}$ (Supplementary Fig. 9) ${ }^{41}$. As expected,

\section{Table 1 The fitted results of recombination rate constants for thermally evaporated perovskite films with different $\mathrm{Cs}_{4} \mathrm{PbBr}_{6}$ concentrations.}

\begin{tabular}{llll} 
Materials & $\boldsymbol{k}_{\mathbf{1}}\left(\mathbf{s}^{-\mathbf{1}}\right)$ & $\boldsymbol{k}_{\mathbf{2}}\left(\mathbf{c m}^{\mathbf{3}} \mathbf{s}^{-\mathbf{1}}\right)$ & $\boldsymbol{k}_{\mathbf{3}}\left(\mathbf{c m}^{\mathbf{6}} \mathbf{s}^{-\mathbf{1}}\right)$ \\
\hline $\mathrm{Cs} / \mathrm{Pb}=1.56$ & $7.14 \times 10^{8}$ & $9.34 \times 10^{-10}$ & $1.83 \times 10^{-27}$ \\
$\mathrm{Cs} / \mathrm{Pb}=1.24$ & $1.69 \times 10^{9}$ & $1.47 \times 10^{-10}$ & $3.16 \times 10^{-28}$
\end{tabular}

The $k_{7}$ is extracted from time-resolved PL under the low excitation fluence, and $k_{2}, k_{3}$ are obtained from the global fitting of recombination rates-carrier density curves.

the $\mathrm{Cs} / \mathrm{Pb}=1.56$ shows a relatively lower defect density of $6.22 \times$ $10^{15} \mathrm{~cm}^{-3}$ compared to $\mathrm{Cs} / \mathrm{Pb}=1.24\left(7.83 \times 10^{15} \mathrm{~cm}^{-3}\right)^{42,43}$.

Moreover, the charge-carrier recombination kinetics can be quantitatively described by the following Eq. 2:

$$
-\frac{d n(t)}{d t}=k_{1} \cdot n(t)+k_{2} \cdot n(t)^{2}+k_{3} \cdot n(t)^{3}
$$

The $k_{2}$ and $k_{3}$ can be extracted by fitting transient absorption (TA) spectra under various excitation fluences with Eq. 2 (Fig. $2 \mathrm{a}-\mathrm{f})^{28,44}$. The fitting results are summarized in Table 1 (calculation details in Supplementary Information and Supplementary Fig. 10). The bi-molecular recombination constant $k_{2}$ of $\mathrm{Cs} / \mathrm{Pb}=1.56$ is about 8 times higher than that of $\mathrm{Cs} / \mathrm{Pb}=1.24$, confirming enhanced radiative recombination rate due to stronger carrier confinement in the sample with higher $\mathrm{Cs}_{4} \mathrm{PbBr}_{6}$ content. As expected, the measured PLQY of $\mathrm{Cs} / \mathrm{Pb}=1.56$ $(40.8 \%)$ is indeed higher than that of the $\mathrm{Cs} / \mathrm{Pb}=1.24(21.5 \%)$ under the moderate excitation fluence. We note that the Auger recombination constant $k_{3}$ of $\mathrm{Cs} / \mathrm{Pb}=1.56$ is also about 6 times higher than that of $\mathrm{Cs} / \mathrm{Pb}=1.24$, which are attributed to the smaller $\mathrm{CsPbBr}_{3}$ nano-inclusions and stronger confinement ${ }^{28}$. In conclusion, the incorporation of the $\mathrm{Cs}_{4} \mathrm{PbBr}_{6}$ phase can not only reduce the trap-assisted recombination rate $k_{1}$ but also significantly increase the bi-molecular recombination rate $k_{2}$, which 
a

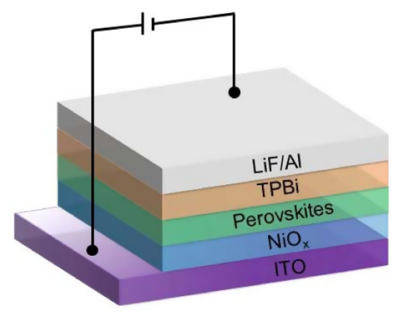

d

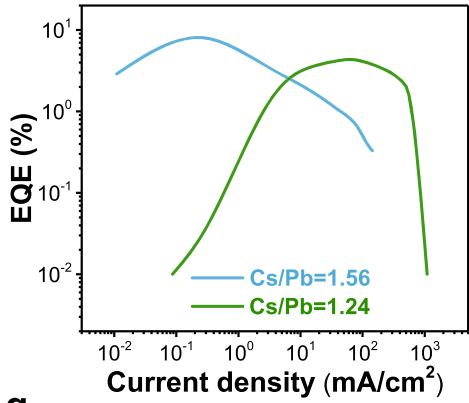

g

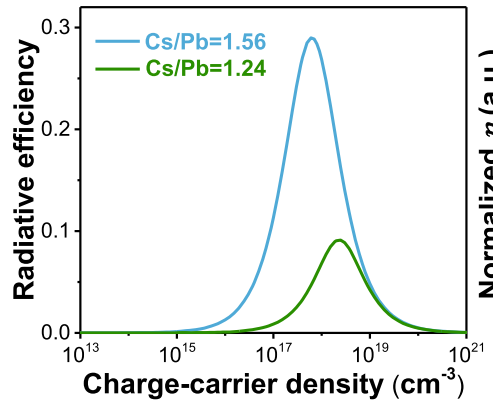

b

h

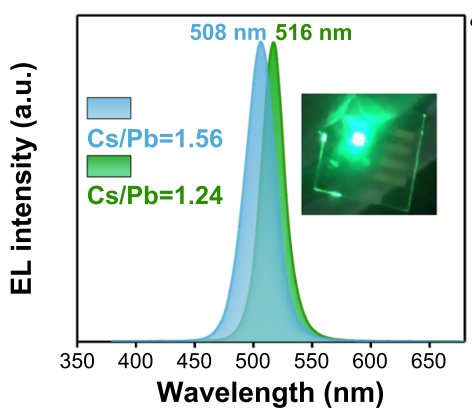

e
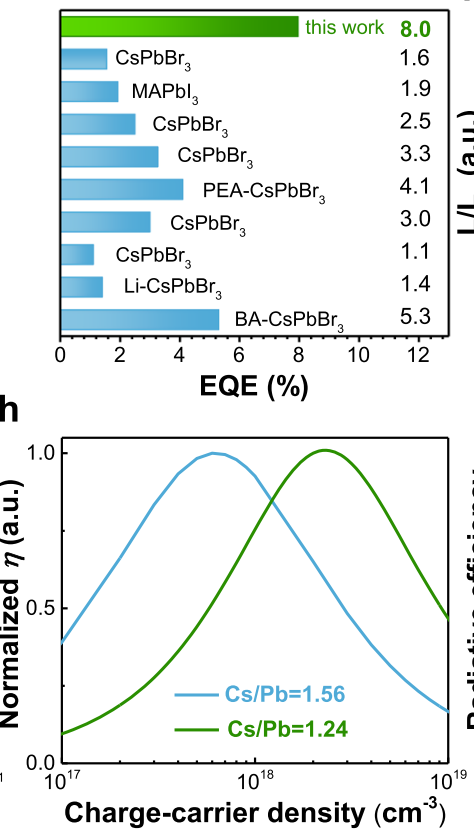

f c
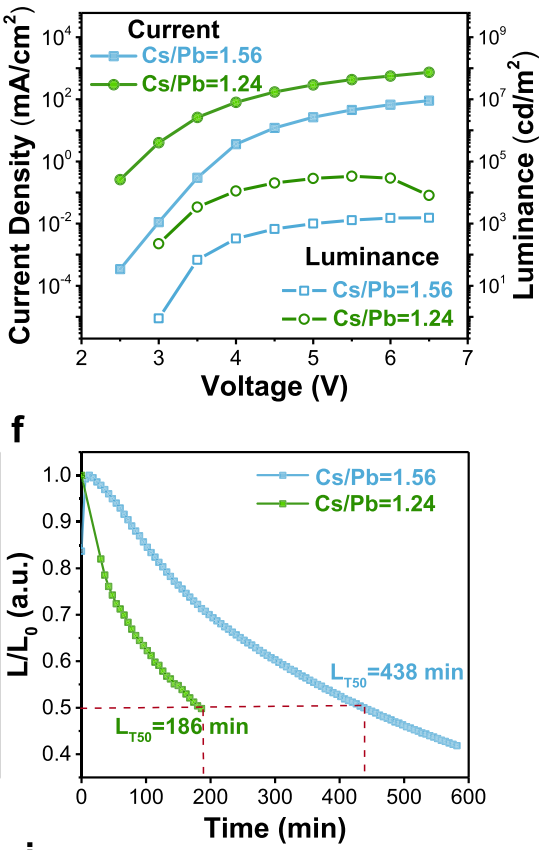

i

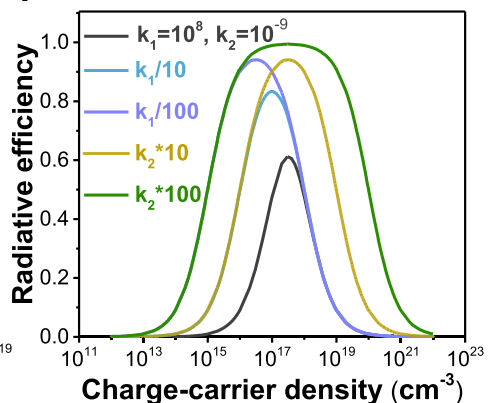

Fig. 3 The performance of Cs-Pb-Br PeLEDs. a The diagram of PeLED structure. $\mathbf{b}$ The normalized electroluminescence spectra of $\mathrm{Cs} / \mathrm{Pb}=1.56 \mathrm{and} \mathrm{Cs} /$ $\mathrm{Pb}=1.24$, the inset is the photo of a Cs/Pb $=1.24 \mathrm{LED}$ driven at $4 \mathrm{~V}$. c Current density and luminance versus voltage characteristics of the PeLEDs. $\mathbf{d}$ EQEcurrent density curves of these two devices. e The EQE summary of thermally evaporated PeLEDs so far reported $25-27,36,51-55$. f Operational stability of devices at an initial luminance of $100 \mathrm{~cd} / \mathrm{m}^{2} . \mathbf{g}$ The simulated radiative efficiency evolution with carrier density by using the fitted recombination constants. $\mathbf{h}$ The normalized radiative efficiency $\eta$ as a function of the carrier density. $\mathbf{i}$ The effect of $k_{1}$ and $k_{2}$ on the radiative efficiency with $k_{3}$ fixed. $k_{1} / 10$ means $k_{1}$ is $10^{7}$ while $k_{2}$ remains invariable $\left(10^{-9}\right)$.

is promising to improve PeLED performance under working conditions (i.e., the electrically injected charge-carrier density is typically lower than that Auger recombination dominates).

The relationship between carrier recombination dynamics and device performance. Encouraged by the enhanced $k_{2}$ and suppressed $k_{1}$ through $\mathrm{Cs}_{4} \mathrm{PbBr}_{6}$ incorporation, we construct thermally evaporated $\mathrm{Cs}-\mathrm{Pb}-\mathrm{Br}$ PeLEDs with a device architecture of indium tin oxide (ITO)/Li doped $\mathrm{NiO}_{x} /$ thermally evaporated perovskites/1,3,5-tris(1-phenyl-1H-benzimidazol-2-yl)benzene (TPBi)/LiF/Al. Figure 3a shows the diagram of our PeLED, where the $\mathrm{Li}$-doped $\mathrm{NiO}_{x}$ serves as a hole transport layer. Note that $\mathrm{Li}$ doped $\mathrm{NiO}_{x}$ is sputtered onto ITO substrate at room temperature to enhance the hole injection of PeLEDs (Supplementary Fig. 11). The uniformity and compactness of our $\mathrm{NiO}_{x}$ layer are characterized by the conductive atomic force microscope (c-AFM), where the conductance mapping shows even-distributed current without significant leakage sites (Supplementary Fig. 12). Figure $3 \mathrm{~b}$ shows the EL spectra of thermally evaporated PeLEDs, with characteristic green emission at 508 and $516 \mathrm{~nm}$, respectively. The blue shift of EL peak for the film of $\mathrm{Cs} / \mathrm{Pb}=$ 1.56 should result from the confinement effect, consistent with the PL spectra. The EL peak positions of the PeLEDs remain constant with increasing applied voltages up to $6.0 \mathrm{~V}$, suggesting the superior color stability of our PeLEDs (Supplementary Fig. 13). Figure $3 c$ plots the current density-voltage-luminance curves of our thermally evaporated PeLEDs, and the current density of PeLEDs with $\mathrm{Cs} / \mathrm{Pb}=1.56$ is lower than that of PeLEDs with $\mathrm{Cs} / \mathrm{Pb}=1.24$, which is attributed to the reduced carrier mobility with higher $\mathrm{Cs}_{4} \mathrm{PbBr}_{6}$ content (Supplementary Fig. 9) ${ }^{45}$. As expected, PeLED with $\mathrm{Cs} / \mathrm{Pb}=1.56$ demonstrates improved device performance of $8.0 \% \mathrm{EQE}$, exceeding that of all previously reported thermally evaporated PeLEDs (Fig. 3e). Supplementary Fig. 14 presents the histogram of the maximum EQE values for 30 devices, which show an average EQE of $6.8 \%$ with a low standard deviation (1.1\%), demonstrating good reproducibility of our thermally evaporated PeLEDs. It appears that the $\mathrm{Cs} / \mathrm{Pb}=1.24$ sample shows relatively severer EQE rolloff, which may be ascribed to charge imbalance caused by different electron/hole mobility (Supplementary Table 3) and Joule heating generated by the larger current density. Operational 
stability is another key parameter for PeLEDs. Operated at an initial luminance of $100 \mathrm{~cd} / \mathrm{m}^{2}$, PeLEDs with $\mathrm{Cs} / \mathrm{Pb}=1.56$ exhibit a superior $\mathrm{L}_{\mathrm{T} 50}$ (indicating the time that the luminance decreased to $50 \%$ of the initial value) of $438 \mathrm{~min}$, which is much longer than that of PeLEDs with $\mathrm{Cs} / \mathrm{Pb}=1.24$ (Fig. 3f). Such operational stability is attributed to the improved efficiency and all-inorganic nature of perovskite film, and this represents one of the most stable thermally evaporated PeLEDs and is comparable with that of solution-processed ones ${ }^{46,47}$.

To provide insights concerning the relationship between charge recombination dynamics and device performance, we compare the EQE-current density curves with the radiative efficiency $(\eta)$-carrier density evolution to investigate the influence of spatial confinement on the LED performance. For the electroexcited PeLED devices, the radiative efficiency $(\eta)$ is positively correlated to the internal quantum efficiency, where radiative efficiency $\eta$ can be calculated based on Eq. 1 using the recombination constants in Table 1 . Figure $3 g$ presents the simulated $\eta$ versus excited carrier density (which is associated with current density), indicating higher peak radiative efficiency of PeLEDs with $\mathrm{Cs} / \mathrm{Pb}=1.56$ compared with that of $\mathrm{Cs} / \mathrm{Pb}=$ 1.24 , which is consistent with the behavior of our PeLEDs. For deep understanding, the maximal radiative efficiency $\left(\eta_{\max }\right)$ can be expressed as Eq. $3^{30}$ :

$$
\eta_{\max }=\frac{1}{1+2 \sqrt{k_{1} k_{3}} / k_{2}}
$$

From Eq. 3, the $\eta_{\max }$ is positively proportional to $k_{2}$ and negatively proportional to $k_{1}$, thereby the LED of $\mathrm{Cs} / \mathrm{Pb}=1.56$ with higher $k_{2}$ and smaller $k_{1}$ should obtain better EQE. Figure $3 \mathrm{~h}$ shows the normalized $\eta$ evolution with carrier density, indicating peak $\eta$ can be obtained at a relatively low carrier density with increasing $\mathrm{Cs}_{4} \mathrm{PbBr}_{6}$. This trend is similar to the EQE-current density curves in Fig. 3d.

Despite great progress achieved in EQE improvement of thermally evaporated PeLEDs, a maximum EQE of $8 \%$ is still lower than that of solution-processed ones. To further propose possible methods for achieving highly efficient thermally evaporated $\mathrm{Cs}-\mathrm{Pb}-\mathrm{Br}$ LEDs, based on the $\mathrm{Cs} / \mathrm{Pb}=1.56$, we modify the $k_{1}$ and $k_{2}$ by one and two orders of magnitude to observe the change of $\eta$-current density curves. As shown in Fig. 3i, at the same order of magnitude, the influence of $k_{2}$ on peak radiative efficiency is more prominent. Meanwhile, the shape of $\eta$-current density curves gradually becomes broader with the increase of $k_{2}$, which indicates it will maintain moderate efficiency over a wider range of carrier density namely improved roll-off. From this perspective, focusing on increasing $k_{2}$ may be a more efficient way to boost device performance. Fundamentally, $k_{2}$ is directly correlated with the wavefunction overlap between electrons and holes, which can be controlled by the spatial confinement effect. For our thermally evaporated $\mathrm{CsPBrr}_{3}$, the incorporation of $\mathrm{Cs}_{4} \mathrm{PbBr}_{6}$ breaks the $3 \mathrm{D}$ connection of $\left[\mathrm{PbBr}_{6}\right]^{4-}$ to provide spatial confinement of electrons and holes, leading to the significant improvement of $k_{2}$. However, the encapsulation of $\mathrm{CsPbBr}_{3}$ by $\mathrm{Cs}_{4} \mathrm{PbBr}_{6}$ is not fully controllable in our thermally evaporated film. As a result, $\mathrm{CsPbr}_{3}$ domains with partial or completely none $\mathrm{Cs}_{4} \mathrm{PbBr}_{6}$ are nearly inevitable, leading to inadequate enhancement of $k_{2}$. Nonetheless, a record EQE of $8.0 \%$ of our thermally evaporated PeLEDs is very encouraging considering the very limited optimization done so far. We believe further optimization towards $>20 \% \mathrm{EQE}$ is in near future.

Demonstration of large-area and patterned PeLEDs. To further demonstrate the uniformity and scalability of the thermal evaporation technique, large-area Cs-Pb-Br film reaching $90 \mathrm{~cm}^{2}$ is achieved for the first time. Figure 4a shows the photograph of asprepared film, indicating uniform PL emission under ultraviolet light irradiation. Furthermore, we measure the PL mapping to characterize the uniformity of our large-area perovskite films. Encouragingly, the as-prepared film shows uniform PL emission with negligible fluctuations not only on the micro-scale $(5 \mu \mathrm{m} \times 5$ $\mu \mathrm{m})$ (Fig. $4 \mathrm{~b}$ ) but also on the macroscopic scale $\left(90 \mathrm{~cm}^{2}\right)$ (Fig. $4 \mathrm{c}$ ), demonstrating the superior uniformity of the thermally evaporated film. Besides, the micro-area distribution of PL peak positions and FWHM also show little fluctuations (Supplementary Fig. 15). In contrast, large PL emission fluctuations, especially at edge areas, are generally found for solution-processed films ${ }^{13}$. Moreover, the morphology and composition uniformity of our large-area films are also characterized. SEM images show identical morphology for the center and edge sites (Supplementary Fig. 16). Meanwhile, the element distribution of $\mathrm{Cs}, \mathrm{Pb}, \mathrm{Br}$ is uniform not only on the micro-scale but also for the whole film (Supplementary Fig. 17 and Supplementary Fig. 18). The great uniformity of our thermally evaporated film lays the foundation for large-area and efficient PeLED devices.

Furthermore, we can easily realize perovskite patterns using fine metal masks, which resemble OLED displays. Figure $4 \mathrm{~d}$ shows the photograph of as-prepared perovskite patterns with a pixel size of $900 \mu \mathrm{m} \times 900 \mu \mathrm{m}$ and pixel diameter of $\sim 100 \mu \mathrm{m}$, exhibiting bright and uniform green emission under ultraviolet light irradiation. Fluorescence microscope characterization further reveals uniform PL emission without any visible defect appeared at each pixel (Fig. $4 \mathrm{e}$ and Fig. $4 \mathrm{f}$ ), and the edge area is clear and sharp. In contrast, the ink-printing perovskite patterns often show nonuniform morphology due to the serious "coffee ring" effect ${ }^{15}$. We believe these results demonstrate the superiority of thermally evaporated techniques for the fabrication of perovskite patterns. The maturity of thermal evaporating technologies and facilities will certainly facilitate the preparation of PeLED displays.

Encouraged by these findings, we further demonstrate largearea PeLEDs on both rigid glass/ITO substrates and flexible PET/ ITO substrates. Figure $4 \mathrm{~g}$ shows the small-area $\left(48 \mathrm{~mm}^{2}\right)$ PeLEDs with a logo of 'HUST', demonstrating the patterning of our thermally evaporated PeLEDs. Figure $4 \mathrm{~h}$ demonstrates a flexible device with good flexibility under working conditions, which shows uniform electroluminescence at a bending angle of about $45^{\circ}$. To further demonstrate the scalability of our thermally evaporated technique, an ultra-large PeLED with a functional area up to $40.2 \mathrm{~cm}^{2}$ was fabricated (Fig. $4 \mathrm{i}$ ), which is the largest one ever achieved in PeLEDs (Supplementary Table 4). We measured the current density-luminance-voltage $(J-V-L)$ and EQE-current density (EQE-J) curves of our PeLED (Fig. 5a, b). Encouragingly, the as-prepared device delivers a peak EQE of $7.1 \%$ with uniform EL emission over the whole working area (Fig. 5c), showing a slight EQE loss of $0.9 \%$ when functional areas are scaled up from $0.05-40.2 \mathrm{~cm}^{2}$. In comparison, the typical EQE of solution processed-PeLED reduces from $20.2-12.1 \%$ when devices scaled up from $0.04-9 \mathrm{~cm}^{2} 14$. We believe the wellcontrolled deposition during the thermal evaporation process and excellent film uniformity over a large area together contribute to the small EQE loss 22 . We noted that such a large area of $40.2 \mathrm{~cm}^{2}$ is comparable with the commercial cellphone screen, highlighting their great potential to be built on a commercial display screen, and $40.2 \mathrm{~cm}^{2}$ is not the limit for thermally evaporated PeLEDs considering 65-inch OLED display panels have already been mass-produced ${ }^{48}$. Figure $5 \mathrm{~d}$ summarizes the performance of large-area PeLEDs fabricated based on various film-coating methods. The EQE of $7.1 \%$ is one of the highest ever reported for PeLEDs with functional areas exceeding $10 \mathrm{~cm}^{2}$. Moreover, our all-vacuum processed strategy also shows excellent 
a

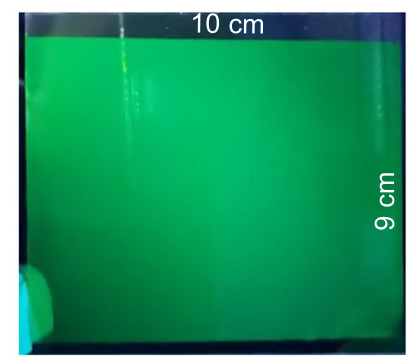

d

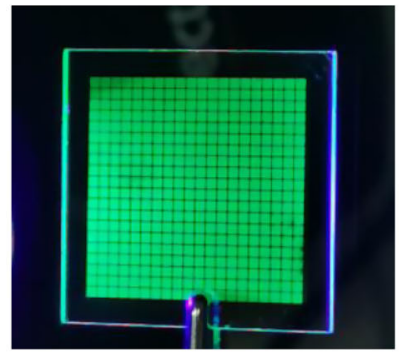

g

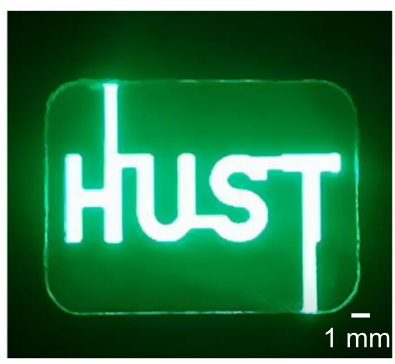

b

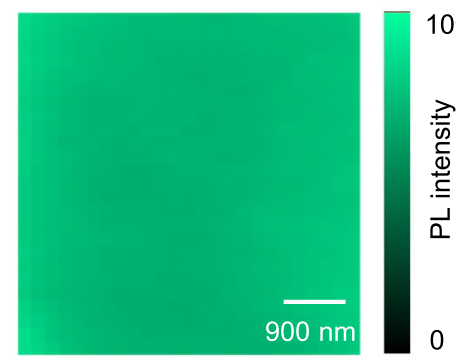

e

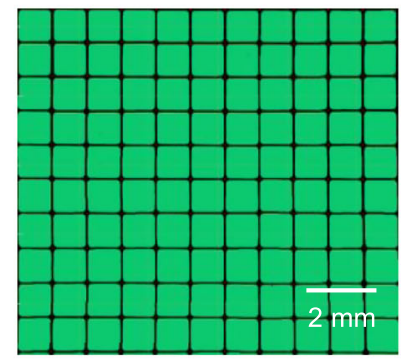

h

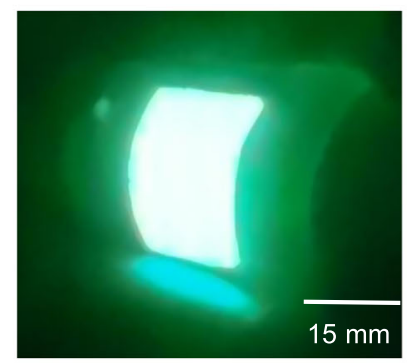

C

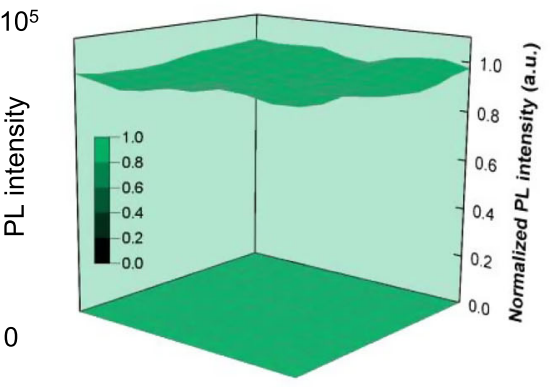

f

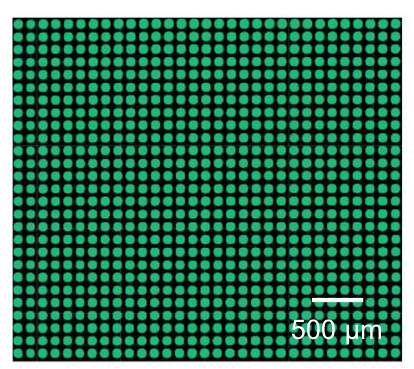

i

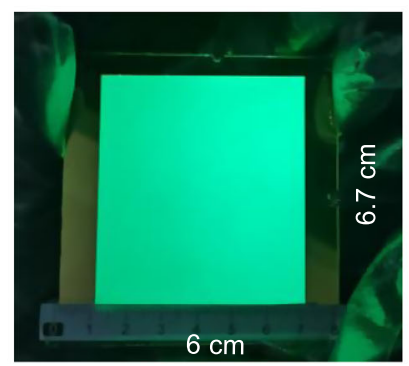

Fig. 4 Potential of thermally evaporated PeLED for display application. a PL image of $90 \mathrm{~cm}^{2} \mathrm{Cs}-\mathrm{Pb}-\mathrm{Br}$ film fabricated by thermal evaporation. b,c The spatial distribution of PL intensity for the micro area $(5 \mu \mathrm{m} \times 5 \mu \mathrm{m})$ and macro area $\left(90 \mathrm{~cm}^{2}\right)$. d The PL photo of $\mathrm{CsPbBr} 3$ film with $900 \mu \mathrm{m} \times 900 \mu \mathrm{m}$ pixels. e, $\mathbf{f}$ The images of fluorescence microscopy characterization for the patterned perovskite film. $\mathbf{g}$ EL photo of PeLED with a "HUST" pattern ( 48 mm²). h A flexible PeLED with an electroluminescent area of $300 \mathrm{~mm}^{2}$. i Photograph $40.2 \mathrm{~cm}^{2}$ PeLED under working conditions.

compatibility with the current OLED mass production line, potentially bringing the commercialization of PeLED technology one step closer.

\section{Discussion}

Slow radiative recombination rate $\left(k_{2}\right)$ is the critical limiting factor faced by three-dimensional perovskite $\mathrm{CsPbBr}_{3}$, which leads to inferior radiative efficiency. In principle, $k_{2}$ is directly correlated with the wavefunction overlap between electrons and holes. Thus, we incorporate zero-dimensional $\mathrm{Cs}_{4} \mathrm{PbBr}_{6}$ into three-dimensional $\mathrm{Cs} \mathrm{PbBr}_{3}$ perovskite for providing spatial confinement of electrons and holes by forming type I heterostructures. As a result, we achieve a bright $\mathrm{Cs}-\mathrm{Pb}-\mathrm{Br}$ film with enhanced $k_{2}$ and suppressed $k_{1}$. When implemented as active layers in LEDs, they showed an impressive EQE of $8.0 \%$, higher than any previously reported thermally evaporated PeLEDs. Taking advantage of the uniformity and scalability of the thermal evaporation technique, we achieve the largest PeLEDs with an EQE of $7.1 \%$ and a functional area up to $40.2 \mathrm{~cm}^{2}$, comparable with commercial cellphone screens. Moreover, the maturity of the thermal evaporation technique facilitates the preparation of perovskite patterns and flexible PeLEDs. Our results demonstrate the great potential of thermally evaporated PeLEDs for nextgeneration display technologies. Further study should continue to improve devices performance by designing better encapsulation of $\mathrm{Cs} \mathrm{PbBr}_{3}$, passivating defects to suppress $k_{1}$, balancing the high PL and efficient carrier injection, and further improving device stability.

\section{Methods}

Materials and chemicals. $\mathrm{CsBr}$ (99.999\%) and $\mathrm{PbBr}_{2}$ (99.999\%) were purchased from Sigma-Aldrich. LiF was purchased from Aladdin Reagent Ltd. TPBi was purchased from Xi'an Polymer Light Technology Corp. Li-doped $\mathrm{NiO}_{\mathrm{x}}$ target ( $\mathrm{NiO}: \mathrm{Li}_{2} \mathrm{O}=$ 98.96:1.04 wt\%) and $\mathrm{Al}$ pellet (99.999\%) were purchased from ZhongNuo Advanced Material Technology Co., Ltd. All chemicals were used as received.

Perovskite film deposition. The $\mathrm{Cs}-\mathrm{Pb}-\mathrm{Br}$ perovskite films with various $\mathrm{Cs} / \mathrm{Pb}$ ratios were fabricated by co-evaporation of $\mathrm{CsBr}$ and $\mathrm{PbBr}_{2}$ in separate crucibles, and we noted that $\mathrm{Cs} / \mathrm{Pb}$ ratios will affect the morphology of $\mathrm{Cs}-\mathrm{Pb}-\mathrm{Br}$ films (details in the Supplementary Note 2). It should be emphasized that the thickness of $\mathrm{CsBr}$ and $\mathrm{PbBr}_{2}$ should be calibrated to obtain the correct scale factor; vacuum higher than $1 \times 10^{-4} \mathrm{~Pa}$ and clean environment of the evaporation chamber is crucial to avoiding pollution of perovskite film, enabling well-performing devices. The films with various $\mathrm{Cs} / \mathrm{Pb}$ ratios are deposited through the evaporation rate adjustment of $\operatorname{CsBr}(1.2-3 \AA / s)$ with a fixed rate of $\operatorname{PbBr}_{2}(1 \AA / s)$ without substrate heating. It is necessary to finely optimize the evaporation rate ratio of $\mathrm{Cs} / \mathrm{Pb}$. According to the previous reports, the high evaporation rate will facilitate the formation of immobile nuclei ${ }^{37}$, the low temperature may impede the growth of nucle $^{36}$, and thereby the combinatorial process is beneficial to produce small grains providing boundaries for the spatial confinement. To accurately control the 
a

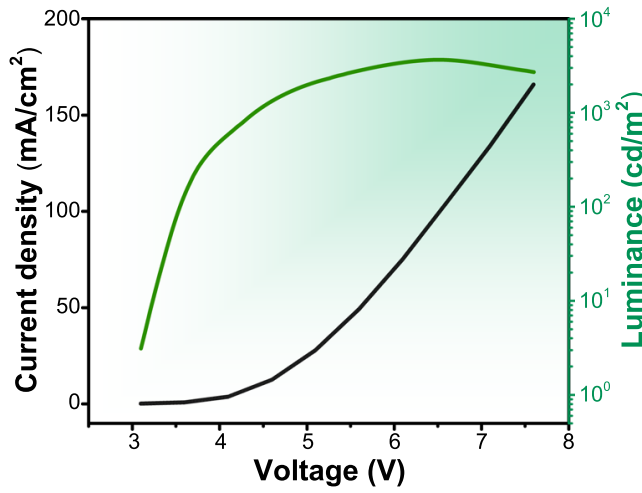

C

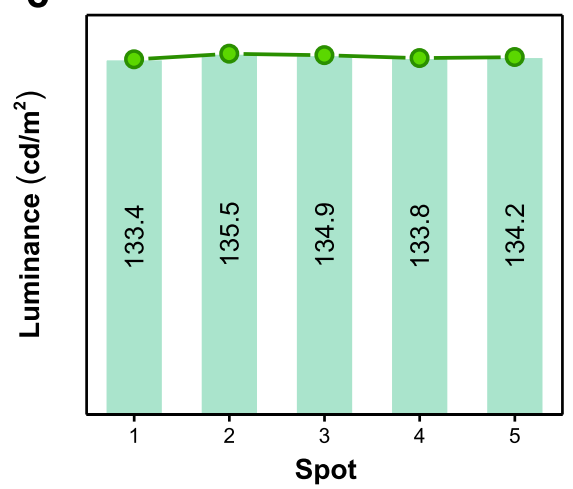

b

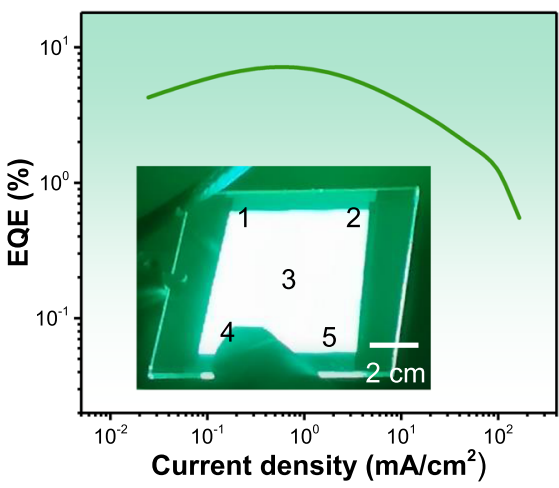

d

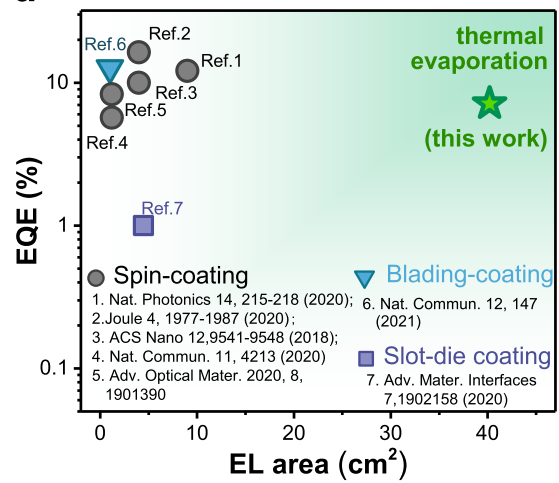

Fig. 5 The performance of large-area PeLEDs. a Current density-voltage-luminance curves. $\mathbf{b}$ EQE-current density curve. c The luminance of spots $1-5$ (corresponding to the marked ones in panel b, inset) for our PeLED. d The reported EQE of large-area PeLEDs (exceed $1 \mathrm{~cm}^{2}$ ) based on various deposition strategies. Clearly, thermal evaporation shows enormous potential for efficient and large-size PeLEDs.

rate, the shutter is open only when both evaporation rates are stable, and the supply powers are kept constant during the whole deposition to minimize fluctuation.

Perovskite LED fabrication. The patterned ITO-coated glass substrates were subsequently cleaned using detergent, acetone, deionized water, and isopropyl alcohol by sonication. Then the ITO substrates were transferred into the sputtering system (JCP500, Beijing Techno Technology Co., Ltd) for depositing Li-doped $\mathrm{NiO}_{\mathrm{x}}(\mathrm{NiO}$ : $\left.\mathrm{Li}_{2} \mathrm{O}=98.96: 1.04 \mathrm{wt} \%\right)$ hole transport layer by magnetron sputtering. The radiofrequency (RF) sputtering power was set at $200 \mathrm{~W}$ without substrate heating, and the flow rate of argon gas was kept at $100 \mathrm{sccm}$. The thickness of $\mathrm{NiO}_{x}$ is about $20 \mathrm{~nm}$. After that, $\mathrm{NiO}_{\mathrm{x}}$ substrates were transferred into the evaporation chamber for perovskite deposition $(80 \mathrm{~nm}$ ). Our thermal evaporator (R27-E, Shenyang Qihui Vacuum Technology Co., Ltd) is connected with a glove box to minimize ambient exposure during sample transferring and device measurements. Finally, TPBi $(17 \mathrm{~nm})$ and LiF/ $\mathrm{Al}$ electrodes $(1 \mathrm{~nm} / 100 \mathrm{~nm})$ were deposited by thermal evaporation under a high vacuum of $<5 \times 10^{-4} \mathrm{~Pa}$. The LED emitting area was $0.05 \mathrm{~cm}^{2}$ as defined by the overlapping area of ITO and Al electrodes. All of the layers were deposited at room temperature. The $40.2 \mathrm{~cm}^{2}$ large-area LED was fabricated with an $\mathrm{Al}$ area of $6.7 \mathrm{~cm} \times$ $6 \mathrm{~cm}$. The "HUST" LED with a total emitting area of $48 \mathrm{~mm}^{2}$ was achieved by the patterned $\mathrm{Al}$ cathode. As for the flexible LEDs, the polyethylene glycol terephthalate (PET) substrates were used for the bending exhibition, while other functional layers are the same as the conventional rigid devices.

Perovskite film and device characterizations. The steady-state PL spectra were recorded in the glovebox using a Photo Research Spectra Scan PR655 under the irradiation of the $365 \mathrm{~nm}$ UV lamp. The ultraviolet-visible absorption spectra were performed on a SolidSpec-3700 UV/374 vis/near-IR spectrophotometer with an integrating sphere. The XRD patterns were recorded with a Philips X'Pert Pro diffractometer with $\mathrm{Cu} \mathrm{K}$ a radiation $(\lambda=1.54 \AA$ ). For transient absorption (TA) measurements, femtosecond laser pulses of an $800 \mathrm{~nm}$ fundamental beam $(5 \mathrm{kHz}$ repetition rate, $35 \mathrm{fs}$ pulse width) were produced using a regenerative amplified $\mathrm{Ti}$ : Sapphire (Legend Duo, Coherent Inc.). Part of the fundamental beam was used to pump an optical parametric amplifier (TOPAS-Prime, Light Conversion) to serve as a narrowband pump, while the other part was focused on a sapphire crystal to generate a white-light supercontinuum probe $(420-750 \mathrm{~nm}$ window with various optical filters). The pump wavelength was $365 \mathrm{~nm}$. Both the pump and probe pulses were directed into a commercial TA spectrometer (Helios, Ultrafast Systems). Delaying the probe pulse relative to the pump provides a time window of up to $8 \mathrm{~ns}$. The instrument response function (IRF) was determined to be $\sim 200 \mathrm{fs}$ by a routine cross-correlation procedure. The transient PL was collected on an Edinburgh Instruments Ltd EPL-370. The spatially resolved PL and temperaturedependent PL were determined using a confocal microscope spectrometer (Alpha300 Raman, WITec) with a $355 \mathrm{~nm}$ laser: the peak intensity maps of largearea films were extracted from the PL spectra of each spot sequentially measured by moving film; the PL position, FWHM, and intensity of micro-area mapping were conducted by automatic stepping machine with a $200 \mathrm{~nm}$ step. The PL images of patterned perovskite film were collected by confocal fluorescence microscopy. The trap densities of the perovskite films were extracted by the dark current-voltage characteristics of the hole-only devices in the device architecture of ITO/ $/ \mathrm{NiO}_{\mathrm{x}} /$ Perovskite/TAPC/ $/ \mathrm{MoO}_{3}(10 \mathrm{~nm}) / \mathrm{Ag}(80 \mathrm{~nm})$ through a computer-controlled Keithley 2400 source meter. The EL performances including current density, luminance, $\mathrm{EQE}$, and operational stability (without encapsulation) of the perovskite LEDs were recorded simultaneously by a commercial measurements system (a programmable Keithley 2400 source meter and a Photo Research Spectra Scan PR655) which was integrated with an $\mathrm{N}_{2}$ filled glovebox. The thermogravimetric analyses of perovskite crystals were conducted on the Diamond TG/DTA (PerkinElmer Instruments) under an $\mathrm{N}_{2}$ atmosphere with a heating rate of $10^{\circ} \mathrm{C} / \mathrm{min}$.

\section{Data availability}

The data that support the plots within this paper and other findings of this study are available from the corresponding author upon reasonable request. The phase diagram of $\mathrm{CsBr}-\mathrm{PbBr}_{2}$ is available at: https://materials.springer.com/isp/phase-diagram/docs/ c_0202533.

Received: 24 February 2021; Accepted: 3 June 2021; Published online: 06 August 2021

\section{References}

1. Cao, Y. et al. Perovskite light-emitting diodes based on spontaneously formed submicrometre-scale structures. Nature 562, 249-253 (2018).

2. Luo, J. et al. Efficient and stable emission of warm-white light from lead-free halide double perovskites. Nature 563, 541-545 (2018). 
3. Liu, Y. et al. Efficient blue light-emitting diodes based on quantum-confined bromide perovskite nanostructures. Nat. Photonics 13, 760-764 (2019).

4. $\mathrm{Li}, \mathrm{Z}$. et al. Modulation of recombination zone position for quasi-twodimensional blue perovskite light-emitting diodes with efficiency exceeding 5\%. Nat. Commun. 10, 1027 (2019).

5. Quan, L. N. et al. Perovskites for next-generation optical sources. Chem. Rev. 119, 7444-7477 (2019).

6. Kumar, S. et al. Ultrapure green light-emitting diodes using two-dimensional formamidinium perovskites: achieving recommendation 2020 color coordinates. Nano Lett. 17, 5277-5284 (2017).

7. Tan, Z.-K. et al. Bright light-emitting diodes based on organometal halide perovskite. Nat. Nanotechnol. 9, 687-692 (2014).

8. Lin, K. et al. Perovskite light-emitting diodes with external quantum efficiency exceeding 20 per cent. Nature 562, 245-248 (2018).

9. Chiba, T. et al. Anion-exchange red perovskite quantum dots with ammonium iodine salts for highly efficient light-emitting devices. Nat. Photonics 12, 681-687 (2018).

10. $\mathrm{Xu}, \mathrm{W}$. et al. Rational molecular passivation for high-performance perovskite light-emitting diodes. Nat. Photonics 13, 418-424 (2019).

11. Du, P., Gao, L. \& Tang, J. Focus on performance of perovskite light-emitting diodes. Front. Optoelectron. 13, 235-245 (2020).

12. Wang, $H$. et al. A multi-functional molecular modifier enabling efficient largearea perovskite light-emitting diodes. Joule 4, 1977-1987 (2020).

13. Chu, S. et al. Large-area and efficient perovskite light-emitting diodes via lowtemperature blade-coating. Nat. Commun. 12, 147 (2021).

14. Zhao, X. \& Tan, Z.-K. Large-area near-infrared perovskite light-emitting diodes. Nat. Photonics 14, 215-218 (2020).

15. $\mathrm{Li}, \mathrm{H}$. et al. Coffee ring elimination and crystalline control of electrohydrodynamically printed high-viscosity perovskites. J. Mater. Chem C. 7, 14867-14873 (2019).

16. Li, D. et al. Inkjet printing matrix perovskite quantum dot light-emitting devices. Adv. Mater. Technol. 5, 2000099 (2020).

17. Zhang, D., Huang, T. \& Duan, L. Emerging self-emissive technologies for flexible displays. Adv. Mater. 32, 1902391 (2020)

18. Geffroy, B., le Roy, P. \& Prat, C. Organic light-emitting diode (OLED) technology: materials, devices and display technologies. Polym. Int. 55, 572-582 (2006).

19. Ávila, J., Momblona, C., Boix, P. P., Sessolo, M. \& Bolink, H. J. Vapordeposited perovskites: The route to high-performance solar cell production? Joule 1, 431-442 (2017).

20. Liu, C., Cheng, Y.-B. \& Ge, Z. Understanding of perovskite crystal growth and film formation in scalable deposition processes. Chem. Soc. Rev. 49, 1653-1687 (2020).

21. Du, P. et al. Vacuum-deposited blue inorganic perovskite light-emitting diodes. ACS Appl. Mater. Interfaces 11, 47083-47090 (2019).

22. Li, J. et al. Highly efficient thermally Co-evaporated perovskite solar cells and mini-modules. Joule 4, 1035-1053 (2020).

23. Liu, X.-K. et al. Metal halide perovskites for light-emitting diodes. Nat. Mater. 20, 10-21 (2021)

24. Kulkarni, S. A., Yantara, N., Tan, K. S., Mathews, N. \& Mhaisalkar, S. G. Perovskite nanostructures: leveraging quantum effects to challenge optoelectronic limits. Mater. Today 33, 122-140 (2020).

25. Li, J. et al. High-throughput combinatorial optimizations of perovskite lightemitting diodes based on all-vacuum deposition. Adv. Func. Mater. 29, 1903607 (2019).

26. Lian, X. et al. Light emitting diodes based on inorganic composite halide perovskites. Adv. Func. Mater. 29, 1807345 (2019).

27. $\mathrm{Hu}, \mathrm{Y}$. et al. Vacuum-evaporated all-inorganic cesium lead bromine perovskites for high-performance light-emitting diodes. J. Mater. Chem. C. 5, 8144-8149 (2017).

28. Chen, Z. et al. Recombination dynamics study on nanostructured perovskite light-emitting devices. Adv. Mater. 30, 1801370 (2018).

29. Xu, L. et al. A bilateral interfacial passivation strategy promoting efficiency and stability of perovskite quantum dot light-emitting diodes. Nat. Commun. 11, 3902 (2020).

30. Xing, G. et al. Transcending the slow bimolecular recombination in leadhalide perovskites for electroluminescence. Nat. Commun. 8, 14558 (2017).

31. Yang, X. et al. Efficient green light-emitting diodes based on quasi-twodimensional composition and phase engineered perovskite with surface passivation. Nat. Commun. 9, 570 (2018).

32. Cho, H. et al. Overcoming the electroluminescence efficiency limitations of perovskite light-emitting diodes. Science 350, 1222-1225 (2015).

33. Kuznetsova, I. Y., Kovaleva, I. S. \& Fedorov, V. A. Interaction of lead bromide with cesium and cadmium bromides. Zh. Neorganicheskoj Khimii 46, 1900-1905 (2001).

34. Akkerman, Q. A., Abdelhady, A. L. \& Manna, L. Zero-dimensional cesium lead halides: history, properties, and challenges. J. Phys. Chem. Lett. 9, 2326-2337 (2018).
35. Quan, L. N. et al. Highly emissive green perovskite nanocrystals in a solid state crystalline matrix. Adv. Mater. 29, 1605945 (2017).

36. Chen, C. et al. Efficient flexible inorganic perovskite light-emitting diodes fabricated with $\mathrm{CsPbBr}_{3}$ emitters prepared via low-temperature in situ dynamic thermal crystallization. Nano Lett. 20, 4673-4680 (2020).

37. Shin, M. et al. Modulation of growth kinetics of vacuum-deposited $\mathrm{CsPbBr} 3$ films for efficient light-emitting diodes. ACS Appl. Mater. Interfaces 12, 1944-1952 (2020)

38. Kang, B. \& Biswas, K. Exploring polaronic, excitonic structures and luminescence in $\mathrm{Cs}_{4} \mathrm{PbBr}_{6} / \mathrm{CsPbBr}_{3}$. J. Phys. Chem. Lett. 9, 830-836 (2018).

39. D'innocenzo, V. et al. Excitons versus free charges in organo-lead tri-halide perovskites. Nat. Commun. 5, 3586 (2014)

40. Ling, Y. et al. Composite perovskites of cesium lead bromide for optimized photoluminescence. J. Phys. Chem. Lett. 8, 3266-3271 (2017).

41. Dong, Y. et al. Bipolar-shell resurfacing for blue LEDs based on strongly confined perovskite quantum dots. Nat. Nanotechnol. 15, 668-674 (2020).

42. Jiang, $\mathrm{Y}$. et al. Reducing the impact of Auger recombination in quasi-2D perovskite light-emitting diodes. Nat. Commun. 12, 336 (2021).

43. Shen, $\mathrm{X}$. et al. $\mathrm{Zn}$-alloyed $\mathrm{CsPbI}_{3}$ nanocrystals for highly efficient perovskite light-emitting devices. Nano Lett. 19, 1552-1559 (2019).

44. Richter, J. M. et al. Enhancing photoluminescence yields in lead halide perovskites by photon recycling and light out-coupling. Nat. Commun. 7, 13941 (2016).

45. Yin, J. et al. Molecular behavior of zero-dimensional perovskites. Sci. Adv. 3, e1701793 (2017).

46. Song, J. et al. Organic-inorganic hybrid passivation enables perovskite QLEDs with an EQE of $16.48 \%$. Adv. Mater. 30, 1805409 (2018).

47. $\mathrm{Wu}, \mathrm{C}$. et al. Alternative type two-dimensional-three-dimensional lead halide perovskite with inorganic sodium ions as a spacer for high-performance lightemitting diodes. ACS Nano 13, 1645-1654 (2019).

48. Matsumoto, E. Development of the OLED mass-production system. SID Symp. Dig. Tech. Pap. 51, 917-920 (2020).

49. Cola, M., Massarotti, V., Riccardi, R. \& Sinistri, C. Binary systems formed by lead bromide with ( $\mathrm{Li}, \mathrm{Na}, \mathrm{K}, \mathrm{Rb}, \mathrm{Cs}$, and $\mathrm{Tl}) \mathrm{Br}$ : a DTA and diffractometric study. Z. f.ür. Naturforsch. A 26, 1328-1332 (1971).

50. Br-Cs-Pb Vertical Section of Ternary Phase Diagram, https://materials. springer.com/isp/phase-diagram/docs/c_0202533.

51. Dänekamp, B. et al. Efficient photo- and electroluminescence by trap states passivation in vacuum-deposited hybrid perovskite thin films. ACS Appl. Mater. Interfaces 10, 36187-36193 (2018).

52. Jia, K. et al. Improved performance for thermally evaporated perovskite lightemitting devices via defect passivation and carrier regulation. ACS Appl. Mater. Interfaces 12, 15928-15933 (2020)

53. Xie, S., Osherov, A. \& Bulović, V. All-vacuum-deposited inorganic cesium lead halide perovskite light-emitting diodes. APL Mater. 8, 051113 (2020).

54. Dumont, A. et al. Extraordinary mass transport and self-assembly: a pathway to fabricate luminescent $\mathrm{CsPBr}_{3}$ and light-emitting diodes by vapor-phase deposition. Adv. Mater. Interfaces 7, 2000506 (2020).

55. Fu, Y. et al. Scalable all-evaporation fabrication of efficient light-emitting diodes with hybrid 2D-3D perovskite nanostructures. Adv. Func. Mater. 30, 2002913 (2020)

\section{Acknowledgements}

This work was financially supported by the National Natural Science Foundation of China (61725401, 62050039, 5171101030, 51761145048, 62004075, 62005089), Fund for Innovative Research Groups of the Natural Science Foundation of Hubei Province (2020CFA034), the National Key R\&D Program of China (2016YFB0700702, and 2016YFB0201204), the Post-Doctoral Innovative Talent Support Program (BX20200142), China Postdoctoral Science Foundation (2020M682413, 2019M662624), and the Graduates' Innovation Fund of Huazhong University of Science and Technology (No. 2021yjscxcy036). The authors from HUST thank the Analytical and Testing Center of HUST and the facility support of the State Key Laboratory of Materials Processing and Die \& Mould Technology. We also thank Prof. Tianyou Zhai from HUST for providing access to conducting optical characterization.

\section{Author contributions}

J. T. and J-J. L. supervised the whole project. P-P. D., J-H. L. and L. W. designed and performed most of the experiments, characterizations, and analysis; L. W. was involved in the EL device fabrication and optimization; L. S. was involved in the large-area device fabrication and characterization; X. W. and W-X. L carried out TA measurements; X. X. and Y. M. provided the optical characterizations; L-B. Y. assisted in device measurement. J-C. P. measured the EDS. J-J. L. organized the outline of this paper, and with P-P. D., JH. L, J. T. wrote the paper; all authors discussed the results and commented on the paper.

\section{Competing interests}

The authors declare no competing interests. 


\section{Additional information}

Supplementary information The online version contains supplementary material available at https://doi.org/10.1038/s41467-021-25093-6.

Correspondence and requests for materials should be addressed to J.L. or J.T.

Peer review information Nature Communications thanks the anonymous reviewer(s) for their contribution to the peer review of this work.

Reprints and permission information is available at http://www.nature.com/reprints

Publisher's note Springer Nature remains neutral with regard to jurisdictional claims in published maps and institutional affiliations. (c) (i) Open Access This article is licensed under a Creative Commons Attribution 4.0 International License, which permits use, sharing, adaptation, distribution and reproduction in any medium or format, as long as you give appropriate credit to the original author(s) and the source, provide a link to the Creative Commons license, and indicate if changes were made. The images or other third party material in this article are included in the article's Creative Commons license, unless indicated otherwise in a credit line to the material. If material is not included in the article's Creative Commons license and your intended use is not permitted by statutory regulation or exceeds the permitted use, you will need to obtain permission directly from the copyright holder. To view a copy of this license, visit http://creativecommons.org/ licenses/by/4.0/.

(C) The Author(s) 2021 